\title{
New Metallicity Independent Constraints of Galactic Infall From Deuterium Observations
}

\author{
Tijana Prodanović*t \\ Department of Physics, University of Novi Sad \\ E-mail: prodanvceif.ns.ac.yu
}

\section{Brian D. Fields}

Department of Astronomy, University of Illinois

E-mail: bdfields@illinois.edu

\begin{abstract}
Local deuterium observations have shown large variations over different lines of sight. However, it has been recently proposed that such variations can be explained by strong depletion of deuterium onto dust grains. Consequently, recent Far Ultraviolet Spectroscopic Explorer (FUSE) deuterium observations represent only a lower bound on the true local deuterium abundance which has thus been estimated to be as high as $\sim 85 \%$ of the primordial $\mathrm{D}$ abundance, as opposed to previous estimates of $\sim 55 \%$. Such high local deuterium abundance could be explained with Galactic infall. Within our analytical model we demonstrate that such high local D abundance in fact requires a significant infall. Our constraint comes from the FUSE deuterium observations AND Galactic gas fraction estimates, which, when used in concert, demand infall rate comparable to the star-formation rate. Moreover, our analysis also constrains the fraction of stellar mass that is returned to the ISM to a range $0.1<R \lesssim 0.4$ which is just marginally consistent with modern initial mass functions. Finally, the requirement of infall is broadly consistent with hierarchical structure formation. Thus, our results offer new qualitative and quantitative ways of placing Galactic evolution in the larger cosmological context.
\end{abstract}

10th Symposium on Nuclei in the Cosmos

July 27 - August 1, 2008

Mackinac Island, Michigan, USA

\footnotetext{
${ }^{*}$ Speaker.

${ }^{\dagger}$ The work of TP is supported in part by the Provincial Secretariat for Science and Technological Development, and by the Republic of Serbia under project number 141002B.
} 


\section{Introduction}

Deuterium is only created in the big bang nucleosynthesis (BBN) while all other processes destroy it $[1,2]$ thus its abundance should monotonically decrease after the BBN. However, measurements of the D abundance in the interstellar medium (ISM) have revealed large variations over different lines of sight, that can be greater than a factor of 3! Based on the recent Far Ultraviolet Spectroscopic Explorer (FUSE) deuterium observations, Linsky et al.(2006) [3] claim that these local variations are due to efficient D depletion onto dust grains. Consequently, Linsky et al.(2006) determine the true ISM deuterium abundance to be $(D / H)_{\mathrm{ISM}+\text { dust }} \geq(2.31 \pm 0.24) \times 10^{-5}$ which is almost at the level of the primordial value. In the context of galactic chemical evolution (GCE), most models need some level of infall of primordial gas in order to explain such high D abundance [4]. In our work, we adopt the new high local D abundance of Linsky et al.(2006) and combine it with a wide range of observed Galactic gas fractions in order to constrain different infall models. We find this approach to be very constraining and in fact, we find that such high D abundance requires an infall rate that almost balances out the star formation rate within preferred GCE models.

\section{Model}

We construct a model for Galactic evolution with infall and no outflow, where infalling material is assumed to be primordial in composition. We adopt the infall rate that is arbitrarily proportional to the star formation rate [5]. This is consistent with results of recent galaxy formation simulations [6]. In such model baryonic mass of the Galaxy thus increases with time as

$$
\dot{M}_{\text {baryon }}=\alpha \psi
$$

where $\psi(t)$ is the star formation rate, while $\alpha$ is the infall proportionality constant. Assuming a complete destruction of $\mathrm{D}$ in stars and defining the ratio of gas at time $t$ to the initial baryonic mass $M_{\text {baryon, } 0}$ as $\mu \equiv M_{\mathrm{ISM}}(t) / M_{\text {baryon, } 0}$, deuterium evolution from its primordial $D_{p}$ to present day value $D(t)$, can be found as a function of the return fraction $R$ - the fraction of stellar mass that is returned to the ISM in the instantaneous recycling approximation, and $\alpha$, as [7]

$$
\frac{D(t)}{D_{p}}=\frac{R}{\alpha+R}\left(\frac{\alpha}{R}+\mu^{\frac{\alpha+R}{1-\alpha-R}}\right)
$$

where $D \equiv X_{D}=\rho_{D} / \rho_{\text {baryon }}$ is the deuterium mass fraction for a given epoch. Another useful observable that is directly sensitive to star formation and infall rates is the present gas mass fraction defined as $\omega \equiv M_{\mathrm{ISM}}(t) / M_{\mathrm{baryon}}(t)$. In our model, it can be expressed as [7]

$$
\omega(t) \equiv \frac{M_{\mathrm{ISM}}}{M_{\text {baryon }}}=\frac{1-R-\alpha}{1-R-\alpha \mu(t)} \mu(t)
$$

where $M_{\text {baryon }} \equiv M_{\text {baryon }}(t)$ is the total baryonic mass at a given epoch. Equations (2.2) and (2.3) now relate observables $D$ and $\omega$ to the model parameters $\alpha$ and $\mu$, which together respectively quantify the current and integrated rates of Galactic infall. Moreover, at late times, $D$ and $\omega$ 
approach minimum values and combine to give the limiting curve above which no solutions can be found. We find the limiting curve to be [7]

$$
\frac{D_{\min }}{D_{p}}=\frac{1}{1+R(1-\omega)(1-R)}
$$

Equation (2.4) includes the return fraction $R$ as a parameter and can thus be used to discriminate between different initial mass functions (IMFs) which all directly determine the value of $R$.

We will find that for only some values of $R$ is it even possible to find a Galactic evolution model which fits both high present-day interstellar deuterium and the observed gas fraction. Thus these observations could provide hints about the IMF, its mass limits, and the physics of stellar gas return. To illustrate the possibilities, we will present our results for the case of $R=0.3$ but also for a range of $R \in(0.1,0.5)$. As we will see, when solutions are possible, uncertainties in $R$ allow a wider infall rate range.

\section{Results}

Before probing the Galactic infall using our model reflected in (2.2) we must first specify the observables and their uncertainties - deuterium and gas fraction observations. Since deuterium should be monotonically decreasing after the Big Bang, it is always useful to compare its local value to the primordial one, which we adopt be $(\mathrm{D} / \mathrm{H})_{\mathrm{p}}=2.75_{-0.19}^{+0.24} \times 10^{-5}$ [8]. For the ISM deuterium abundance we use a range of measured gas-phase deuterium abundances $(\mathrm{D} / \mathrm{H})_{\mathrm{ISM}}=$ $(0.5-2.2) \times 10^{-5}[3]$ with the central value of $(\mathrm{D} / \mathrm{H})_{\mathrm{ISM}}=(1.56 \pm 0.04) \times 10^{-5}$ [9]. Finally, for a new estimated ISM deuterium abundance that corrects for significant depletion onto dust we take the value of $(\mathrm{D} / \mathrm{H})_{\mathrm{ISM}+\text { dust }} \geq(2.31 \pm 0.24) \times 10^{-5}$ [3]. Since the chemical evolution formalism most naturally uses mass fraction as an abundance measure we transform these $\mathrm{D} / \mathrm{H}$ ratios to mass fractions and find the ratios of ISM-to-primordial deuterium mass fractions to be [7]

$$
\begin{aligned}
\frac{D_{\text {ISM }}}{D_{\mathrm{p}}} & =0.53_{-0.36}^{+0.22} \\
\frac{D_{\text {ISM }+ \text { dust }}}{D_{\mathrm{p}}} & =0.78_{-0.10}^{+0.11}
\end{aligned}
$$

For the present Galactic interstellar gas mass we adopt a full range of observed values $[10,11]$

$$
\omega_{\mathrm{obs}} \equiv\left(\frac{M_{\mathrm{ISM}, \mathrm{MW}}}{M_{\mathrm{baryon}, \mathrm{MW}}}\right)_{\mathrm{obs}} \sim 0.07-0.30
$$

where even with these significant uncertainties, we can places strong constraints on the infall rate $\alpha$. Finally, the return fraction $R$, i.e. the mass fraction of stellar population that is returned to the ISM, can be directly calculated from the IMF. Adopting the standard Salpeter IMF [12] we find $R \approx 0.30$ [7], which, to the first decimal, does not change with changing mass ranges of a given stellar population. Adopting a more realistic IMF [13] that is flatter in the higher-mass regime yields a larger return fraction closer to $R \sim 0.4$. Our results are presented on Figure 1 [7]. The left panel shows deuterium mass fraction as a function of the gas mass fraction (2.2), for adopted retun fraction $R=0.3$. The curves that go through the overlap region between the observed $\mathrm{D}$ and 
gas fraction bands reflect the observationally allowed parameters of chemical evolution models. These curves are colored in red and we see that infall is required and in fact almost comparable to the star formation rate with the infall parameter $0.5 \lesssim \alpha \lesssim 1$. On the right panel of Figure 1 we plot the limiting curves (2.4) above which no solution can be found, for a range of return fractions $0.1 \leq R \leq 0.5$. Overlap ranges between observed $D$ and $\omega$ are presented as boxes. We see that the only return fractions that are allowed are $0.1<R \lesssim 0.4$.
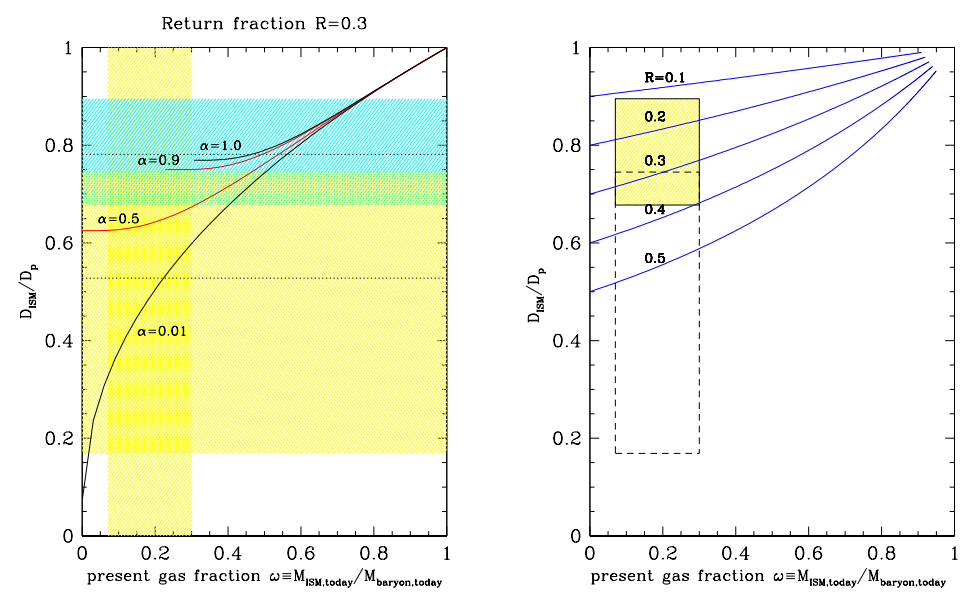

Figure 1: Left panel: The ratio of the total present-day to primordial D mass fraction $D_{\mathrm{ISM}} / D_{p}$ as a function of the present gas mass fraction $\omega$, for assumed return fraction $R=0.3$. Dot-dashed black line along with the top (cyan) band corresponds to the ratio of the D mass fractions for the ISM D measured by FUSE and corrected for depletion onto dust [3] and primordial D [8] as given in (3.2). Bottom (yellow) band with the central value of (3.1) [9] given as a dot-dashed black line corresponds to scatter of D abundances observed along different lines of sight. Vertical (yellow) band reflects the observed range of present gas mass fraction $\omega_{\mathrm{obs}}$ from (3.3). Different solid curves correspond to infall rates with proportionality constant: $\alpha=0.01,0.5,0.9,1.0$. Right panel: The ratio of the limiting present-to-primordial D mass fraction $D_{\text {ISM }} / D_{p}$ as a function of the limiting gas mass fraction $\omega$. The boxes present the overlap regions between the observed range the of present gas mass fraction $\omega_{\mathrm{obs}}$ (3.3) and measured ISM D abundances with (top, yellow box) and without (bottom, dashed box) depletion onto dust [3].

\section{Conclusion}

New observations of the local D abundance have revealed that the true D abundance, once corrected for the depletion onto dust grains, could be very close the primordial value [3], which in most GCE models can be explained by having some level of infall of (close to) pristine gas that would replenish the Galactic deuterium. In this work we have shown that using together the observations of local deuterium abundance and the gas mass fraction constrains the infall rate rather strongly and can thus be used to discriminate between different GCE models. Specifically, we have found that local $\mathrm{D}$ and gas mass observations demand significant infall rate of pristine material with the proportionality constant $0.5 \lesssim \alpha \lesssim 1$ [7]. This is consistent with hierarchical assembly of galaxies by accretion. As an input, our model requires the return fraction $R$ to be set, and is thus, 
also constraining of the adopted IMF which directly determines the value of $R$. We have found that modern IMFs [13] which demand high return fractions $R \sim 0.4$ are just marginally allowed by observations (Figure 1, right panel). Finally we note that if the local D is so severely depleted onto dust as suggested by Linsky et al.(2006) [3], one could worry that high-redshift QSO observations may suffer from a similar effect, however in this case, the standard BBN theory in the light of 5year WMAP data [14] could allow for only a small upward correction to the deuterium abundance due to dust depletion, before breaking the concordance between theoretically predicted primordial and observed high-redshift deuterium abundance [15].

\section{References}

[1] Epstein R. I., Lattimer J. M. and Schramm D. N., The origin of deuterium, Nature 263198 (1976)

[2] Prodanović T. and Fields B. D., On Nonprimordial Deuterium Production by Accelerated Particles, Astrophys. J. 59748 (2003) [astro-ph/0307183]

[3] Linsky J. L. et al., What Is the Total Deuterium Abundance in the Local Galactic Disk?, Astrophys. J. 6471106 (2006) [astro-ph/0608308]

[4] Romano D., Tosi M., Chiappini C. and Matteucci F., Deuterium astration in the local disc and beyond, Mon. Not. R. Astron. Soc. 369295 (2006) [astro-ph/0603190]

[5] Larson, R. B., Cosmogeny-Effect of infalling matter on galactic heavy element content, Nature 2367 (1972)

[6] Kereš D., Katz N., Weinberg D. H., and Davé R., How do galaxies get their gas?, Mon. Not. R. Astron. Soc. 3632 (2005) [astro-ph/0407095]

[7] Prodanović T. and Fields B. D., FUSE Deuterium Observations: A Strong Case For Galactic Infall, Journal of Cosmology and Astro-Particle Physics 09003 (2008) [arXiv:0804.3095]

[8] Cyburt R. H., Fields B. D. and Olive K. A., Primordial nucleosynthesis in light of WMAP, Phys. Lett. B 567227 (2003) [astro-ph/0302431]

[9] Wood B. E., Linsky J. L., Hébrard G., Williger G. M., Moos H. W. and Blair W. P., Two New Low Galactic D/H Measurements from the Far Ultraviolet Spectroscopic Explorer, Astrophys. J. 609838 (2004) [astro-ph/0403606]

[10] Holmberg J. and Flynn C., The local surface density of disc matter mapped by Hipparcos, Mon. Not. R. Astron. Soc. 352440 (2004) [astro-ph/0405155]

[11] Flynn C., Holmberg J., Portinari L., Fuchs B. and Jahreiß H., On the mass-to-light ratio of the local Galactic disc and the optical luminosity of the Galaxy, Mon. Not. R. Astron. Soc. 3721149 (2006) [astro-ph/0608193]

[12] Salpeter, E. E. The Luminosity Function and Stellar Evolution, Astrophys. J. 121161 (1955)

[13] Baldry I. K. and Glazebrook K., Constraints on a Universal Stellar Initial Mass Function from Ultraviolet to Near-Infrared Galaxy Luminosity Densities, Astrophys. J. 593258 (2003) [astro-ph/0304423]

[14] Dunkley J., et al., Five-Year Wilkinson Microwave Anisotropy Probe (WMAP) Observations: Likelihoods and Parameters from the WMAP data, accepted to Astrophys. J. Suppl. (2008) [arXiv:0803.0586]

[15] Cyburt R. H., Fields B. D. and Olive K. A., An update on the big bang nucleosynthesis prediction for 7Li: the problem worsens, Journal of Cosmology and Astro-Particle Physics 1112 (2008) 